Jutta Toelle

\title{
Ideen zum Narrativ von Mission durch Musik in Briefen und Berichten europäischer Missionare in Lateinamerika
}

Im Jahr 1757, wohlgemerkt kurz vor der Ausweisung der Jesuiten aus den spanischen und portugiesischen Kolonialgebieten und der Aufhebung des Ordens 1773, veröffentlichte der französische Jesuit Pierre Francois Xavier de Charlevoix (1682-1761) sein Werk Histoire du Paraguay. Charlevoix, der vor allem durch seine Reisen durch Nordamerika und den Bericht darüber berühmt geworden war, ${ }^{1}$ lieferte in seinem letzten Werk, ${ }^{2}$ das auf verschiedenen Schriften von Missionaren basierte, eine wunderbare Darstellung des Narrativs von Mission durch Musik, aus mindestens zweiter Hand und, zeitlich gesehen, am Ende des Narrativs:

»Indem die Jesuiten auf den Flüssen hinfuhren, so wurden sie gewahr, daß, wenn sie sich auf eine heilige Art die Zeit zu vertreiben, geistliche Lieder sungen, ganze Haufen Indianer herbey liefen, ihnen zuzuhören, und einen ganz besondern Gefallen daran zu haben schienen. Sie machten dieses sich zu nutze, indem sie ihnen erklärten, was sie sängen; und es war eben, als wenn diese Gesänge ihre Herzen verändert, und sie zu den Gesinnungen, die sie ihnen beybringen wollten, geneigt gemacht hätten. Denn sie hatten gar nicht viele Mühe sie zu überreden, ihnen zu folgen; sie fanden sie gelehrig, und nach und nach brachten sie ihren Gemüthern die erhabensten Begriffe von der Religion bey. Auf solche Art machten sie in diesen wilden Ländern dasjenige wircklich wahr, was die Fabel von dem Orpheus und Amphion erzählt.« $\ll^{3}$

Dieses Beispiel für das sehr romantisierte Bild der musikalischen Begegnung zwischen Europäern und Amerikanern, mit Anklängen an populäre Ideen des bon sauvage, wurde weitergegeben und immer wieder kopiert. ${ }^{4}$ In Ángel Santos

1 Pierre Francois Xavier de Charlevoix, Histoire et description générale de la Nouvelle France, avec le Journal historique d'un voyage fait par ordre du roi dans l'Amérique septentrionale, Nyon 1722.

2 Pierre Francois Xavier de Charlevoix, Histoire du Paraguay, Paris 1757.

3 Ebda., hier Zitat aus der deutschen Übersetzung (Geschichte von Paraguay und dem Missionswerke der Jesuiten in diesem Lande aus dem Französischen des P. Franz Xaver de Charlevoix, Nürnberg 1768), V, S. 365.

4 Siehe auch den Verweis auf diese Stelle bei Johannes Meier, »Zur Bedeutung der Musik in den amerikanischen Missionen der Jesuiten«, in: Kirchenmusik zwischen Säkularisation und Restauration, hrsg. von Friedrich Wilhelm Riedel, Sinzig 2006, S. 73-90: S. 76. 
Hernández’ Buch Los jesuitas en América (Madrid 1992) ist aus den geistlichen Lieder bereits Instrumentalmusik auf einer Flöte geworden:

»Sie fuhren in ihren kleinen Kanus die Flüsse hinunter und spielten manchmal die Flöte, um sich von ihren Mühen zu erholen; und so sahen sie aus dem Wald eine Vielzahl von Indios auftauchen, die durch jene für sie so fremden Melodien angezogen wurden. Sie [die Indios] folgten ihnen [den Missionaren] eine lange Zeit am Ufer entlang und etablierten so einen ersten Kontakt mit den Missionaren. $\ll^{5}$

In Roland Joffés Film The Mission von 1988, dessen Musik von Ennio Morricone komponiert wurde, wurde dieses Bild zur Einstiegsszene des Films. Die Flöten der müden Missionare wurden zu einer Oboe, »einem zeittypischen Instrument des 17.Jahrhunderts«, wie der Komponist in einem Interview äußerte. ${ }^{6}$ Im Film klingt die Oboe des Missionars kurz nach seinem gefährlichen Aufstieg entlang tosender Wasserfälle wunderbar rein und eingespielt; dass die Oboe ein extrem empfindliches Instrument ist, bleibt unerwähnt. In dieser Szene schauen die ersten fremd und anders aussehenden Männer, offensichtlich angelockt durch die Klänge, zaghaft und vorsichtig hinter den Bäumen hervor, bis sie schließlich ihre Scheu überwinden und sich dem Missionar nähern. Schließlich wird diesem eine Hand ausgestreckt, und das Idyll des gemeinsamen Musikmachens beginnt.

Im Zentrum des vorliegenden Artikels steht die Genese und die Geschichte des Narrativs von Mission durch Musik; ich stütze mich dabei vor allem auf Chroniken, Berichte und Briefe von Missionaren, die im Kontext des spanischen Kolonialreiches in vielen Fällen die einzigen Überlieferungen zu Themen wie der Missionsarbeit vor Ort, zur Art der Begegnung zwischen Europäern und Indigenen, zwischen Christen und Nichtchristen, zwischen Missionaren und Katechumenen sind.

Sorgfältig muss Schicht für Schicht abgeschält werden, um den tatsächlichen Diskurs beurteilen zu können. Einmal mehr wird deutlich, dass - wie Hayden White es formuliert hat - Geschichte keine bloße Sammlung von Fakten ist, die von der Historikerin arrangiert werden, sondern immer eine Form des Diskurses, der auf konventionellen narrativen Formen und der Vorstellungskraft beruht.

5 »Navegaban por los ríos en sus pequeñas canoas, tocando a veces la flauta para alivio de sus fatigas, y veían aparecer, saliendo del bosque, cantidad de indios atraídos por aquéllas, para ellos tan extrañas melodías. Los seguían largos trechos por la orilla, hasta entablar así un primer contacto con los misioneros.« Ángel Santos Hernández, Los jesuitas en América, Madrid 1992, S. 299.

6 Helga de la Motte-Haber fügte hinzu, dass die Oboe zudem »ein praktisches Instrument [sei], man kann es leicht in einen Sack stecken und dann doch durch einen Urwald spazieren.« DVD The Mission, DVD 2 mit Extras, Interview mit Helga de la Motte-Haber, 5’10-5’27. 
»Auch Klio dichtet«, heißt es, ${ }^{7}$ und: »there is no true story to tell«. Nehmen wir das Narrativ als diskursive Sinnproduktion wahr, erlaubt dies auch »to account $[\ldots]$ for the interest that dominant social groups have $[\ldots]$ in controlling what will pass for the authoritative myths of a given cultural formation. $\ll^{8}$ In unserem Fall ist also zu beachten, dass die franziskanischen und jesuitischen Missionare zwar die Akteure der Missionspraxis waren, die sich die sinnliche Erfahrbarkeit und Verwendbarkeit von Klängen vor Ort zunutze machten, zugleich aber auch die Autoren der wichtigsten Quellen von Mission durch Musik - denn autochthone Quellen gibt es fast keine.

Mit der Etablierung des Narrativs von Mission durch Musik entwarfen die Missionare eine ganz eigenständige Sound history ihrer Arbeit: ein gleichzeitig frühneuzeitlich gebundenes und bis heute beliebtes Narrativ von Klanggeschichte. Denn es geht hier nicht lediglich um Musikgeschichte: es geht um die Hegemonie bestimmter Klänge, um Glocken und Stimmen und um europäische Musik als Teil eines soundscapes der Neuen Welt. ${ }^{9}$ Die Herausforderung besteht mithin darin, das Narrativ in seiner diskursiv-strategischen Kraft ernst zu nehmen, gleichzeitig aber seine spezifischen, im kolonialen Kontext des spanischen Imperiums verankerten Strukturen freizulegen: Wie ist es zu einem derart wichtigen Bestandteil der Missionsgeschichtsschreibung der Frühen Neuzeit geworden? Beziehungsweise, andersherum gefragt: Wie konnte eine offensichtlich recht erfolgreiche Missionierungsidee in ihrer eindimensionalen Zuspitzung zu einem solch langlebigen Narrativ werden? ${ }^{10}$

Dass Musik grundsätzlich ein effektvolles Mittel zur Missionierung war, ist und sein kann, bestreitet niemand. Schon die von den Franziskanern und Dominikanern im Italien des 14. und 15. Jahrhunderts gegründeten Laudesi-Bruderschaften verfolgten das Ziel, durch Laude-Singen das Andacht-Halten zu fördern. Sicher ist auch, dass die Einführung europäischer Kirchenmusik in den Reduktio-

7 »Auch Klio dichtet oder Die Fiktion des Faktischen. Studien zur Tropologie des historischen Diskurses« heißt Hayden Whites Buch Tropics of Discourse: Essays in Cultural Criticism, Baltimore 1978 auf Deutsch.

8 Hayden White, The Content of the Form. Narrative Discourse and Historical Representation, Baltimore 1987, Vorwort, S. X.

9 Siehe hierzu auch meinen Artikel »Mission Soundscapes: Demons, Jesuits and Sounds in Antonio Ruiz de Montoya's Conquista Espiritual (1639)«, in: Empire of the Senses: Sensory Practices and Modes of Perceptions in the Atlantic World, hrsg. von Daniela Hacke und Paul Musselwhite, Leiden 2018.

10 Diese Thematik habe ich in einem 2015 publizierten Artikel eingehender untersucht, siehe Jutta Toelle, »Da indessen die Mohren den Psalm: Lobet den Herrn alle Heiden! abgesungen.< Musik und Klang im Kontext der Mission im México der Frühen Neuzeit«, in: Historische Anthropologie, Thema: Sound, 22/3 (2014), S. 334-349. 
nen unter den Guaraní und anderen Völkern im Rio de la Plata-Becken, als Begleiterscheinung der Einführung von europäischen Werten und religiösen Überzeugungen, auf günstigen Bedingungen aufbaute und sehr erfolgreich war. Hier diente diese Musik nicht nur als Medium der Kommunikation, Alphabetisierung und Konversion, sondern wurde auch zur Rhythmisierung von Tag und Jahr, zur Beschäftigung und Unterhaltung der Indigenen eingesetzt. Diese ex nibilo implantierte, europäisierte Leitkultur ging in den Reduktionen einher mit einer völligen Verleugnung indigener Musik. Angst und Abscheu vor den alten Göttern sowie die Verdammnis der indigenen Feste und der damit verbundenen Gelage machten das Anerkennen einer prähispanischen, vorchristlichen Musik unmöglich, und zudem verbesserten die vertrauten europäischen Klänge den soundscape für die Missionare entscheidend.

Dennoch ist Johannes Meiers Diktum »Die Erfolgsgeschichte der Jesuitenmissionen ist ohne die Musik undenkbar «, ${ }^{11}$ das sich ausschließlich auf die Societas Jesu bezieht, einseitig, denn das Narrativ stammt von den Franziskanern: Das erstmalige Aufscheinen einer Idee »spiritueller Eroberung« der lateinamerikanischen Indigenen auf dem Weg über die Musik findet sich schon in den frühen Briefen der ersten franziskanischen Missionare in Mexico, die 1523 und 1524 in der zerstörten Hauptstadt Tenochtitlán ankamen. Sie berichteten, dass sie die einheimischen Kinder »tagsüber im Lesen, Schreiben und Singen, abends im Predigen und in der christlichen Doktrin $\aleph^{12}$ unterrichteten, und dass das Ziel des Unterrichts das »Lesen, Schreiben, einstimmige [canto llano] und mehrstimmige [canto de órgano] Singen, sowie das Rezitieren der Stundengebete, das Feiern von Messen und das Einüben aller guten christlichen und religiösen Sitten [ynponerlos en todas buenas costumbres christianas y religiosas] $\ll^{13}$ sei.

11 Johannes Meier, »Zur Bedeutung der Musik« (wie Anm. 4), S. 76.

$12 »$ Mi oficio es predicar y enseñar día y noche. En el día enseño a leer, escribir y cantar; en la noche, doctrina cristiana y sermones«. Brief von Pedro de Gante an seine Mitbrüder in Flandern, 1529, in: Ernesto de la Torre Vilar, Fray Pedro de Gante. Maestro y civilizador de América y la Doctrina Cristiana en lengua mexicana de 1553, México D. F. 2001, Anhang, S. 42. Drei Jahre später beschrieb er dasselbe in einem Brief an Kaiser Karl V., ebda., S. 44; siehe auch Emilio Ros-Fábregas, »Imagine all the People... : Polyphonic Flowers in the Hands and Voices of Indians in 16th-century Mexico«, in: Early Music 40/2 (2012), S. 177-189: S. 179.

13 Brief von Martín de Valencia an Karl V., 1532, in: Biblioteca de Autores Españoles 264, Cartas de Indias, I, Religiosos, IX, Madrid 1877, S. 56; siehe auch Gary Tomlinson, The Singing of the New World. Indigenous Voice in the Era of European Contact, Cambridge 2007, S. 176. 
Orientiert an der traditionellen aztekischen Ausbildung der Oberschicht, ${ }^{14}$ unterrichteten die wenigen Franziskaner in Tenochtitlán vor allem einige Söhne der Führungsschicht und bildeten sie zu Multiplikatoren aus. Wenn diese Jugendlichen sonntags ins Land hinauszogen und predigten, sangen sie dabei, wie Jerónimo de Mendieta in seiner Historia Ecclesiastica Indiana berichtet, eine Zusammenfassung christlicher Lehrsätze auf Nahuatl, gefasst in einen »[einstimmigen] sehr angenehmen Choral, der als gute Werbung diente, um Menschen anzuziehen, die ihn lernen wollten ${ }^{15}{ }^{15}$ Wie diese christlichen Gesänge auf Nahuatl aussahen, ist nur in Ansätzen überliefert, doch es gab sie: eine Form christianisierter, rituell an das indigene Alte wie auch das christlich-europäische Neue gebundene, performative Kunst. Die wenigen erhaltenen Handschriften mit christlichen Inhalten, die nach der Eroberung von Azteken auf Nahuatl, aber unter der Ägide der Franziskaner verfasst wurden, erwähnen indigene Instrumente wie die Trommeln huehuetl und teponaztli, sodass davon ausgegangen wird, dass diese zumindest im 16. Jh. auch innerhalb der christlichen Zeremonien verwendet wurden. ${ }^{16}$

Bereits bei dem Franziskaner Toribio de Benavente, der sich in Amerika Motolinía nannte, lässt sich der kalkulierte Einfluss sinnlicher, musikalischer Mittel heraushören. Er berichtet, dass die franziskanischen Missionare den Indigenen die Gebete und die zehn Gebote »in gesungener Form, [...] in ihrer Sprache und in einer anmutigen Melodie « gegeben hätten, »damit sie sie [die Gebete und Gebote] besser schluckten und auch einen Geschmack dabei verspürten $\ll .{ }^{17}$ Der erste Erzbischof von Mexico, der Franziskaner Juan de Zumárraga, schrieb in einem

14 »Como al tono de lo antiguo criábamos á los hijos de los principales dentro de nuestras escuelas, y á los hijos de los plebeyos, lo enseñábamos en el pátio la doctrina cristiana.«, Bernardino de Sahagún, Historia general de las Cosas de Nueva España, hrsg. von Carlos Bustamante, III/10: Relación del Autor digna de ser notada, México 1830, S. 77.

$15 »$ Un canto llano muy gracioso que sirvió de un buen reclamo para atraer gente a la deprender«, Jerónimo Mendieta, Historia Ecclesiastica Indiana, Barcelona 2011, III/19, S. 233. Das Werk von Mendieta wurde 1596 vollendet, im 19. Jahrhundert wiederentdeckt und 1945 erstmals von Salvador Chávez Hayhoe ediert (Fray Jeronimo de Mendieta, Historia eclesiástica indiana. Reimpresión por Salvador Chávez Hayhoe, México 1945). Siehe dazu auch Sergio Botta, »I cantores nella chiesa francescana della nueva Spagna. La costruzione di un sistema di mediazione«, in: La musica dei semplici. L'altra controriforma, hrsg. von Stefania Nanni, Rom 2012, S. 311-318: S. 315-316.

$16 \mathrm{Zu}$ den cantares von Pedro de Gante s. Tomlinson, The Singing of the New World (wie Anm. 13), S. 60; grundsätzlich dazu auch Olivia Bloechl, Native American Song at the Frontiers of Early Modern Music, Cambridge 2008.

$17 »$ Diéronles cantado el Per Signum Crucis, Pater Noster, Ave Maria, Credo y Salve Regina, con los mandamientos en su lengua, de un canto llano gracioso«/ »para que mejor lo tomasen y sintiesen algún sabor«, Motolinía, Memoriales de Fray Toribio de Motolinia: manuscrito de la colección del Señor Don Joaquin García Icazbalceta, hrsg. von Luis García Pimentel, Madrid 1903, Kap. XIII, S. 29. 
Brief an Karl V. aus dem Jahre 1540 über die außerordentliche Wirkung des Gesangs auf die Indigenen:

»Die Geistlichen, die ihnen die Beichte abnehmen, erzählen uns, dass sie sich eher durch die Musik bekehren lassen als durch die Predigten, und wir sehen sie von weither kommen, um Musik zu hören. ${ }^{18}$ Und der Mestize Diego de Valadés (1533-1582), Sohn eines Generals von Hernán Cortés und einer indigenen Frau, verglich die musikalische Pracht der Gottesdienste mit Milchnahrung für Säuglinge: »Vor allem werden die Herzen der Ungläubigen durch solche [musikalisierten] Riten gerührt, und die Seelen dieser neuen Christen fühlen sich sehr bestätigt und gefangen durch diese Äußerlichkeiten; sie sind zudem noch klein [im Glauben], sodass man sie mit Milch ernähren muss und nicht mit festem Essen. «19

Auch König Philipp II. selbst war von der Wirkungsmacht der Musik vor allem auf sich der Bekehrung widersetzende Indigene überzeugt: In den Ordenanzas von 1573, die sich auf das gesamte Kolonialreich bezogen, ordnete er an, wie man sich Menschen nähern solle, die die christliche Botschaft des Friedens nicht annehmen wollten: »Um mehr Bewunderung und Aufmerksamkeit unter den Ungläubigen zu erzeugen, können sie, wenn ihnen dies angemessen erscheint, Musik von Sängern und verschiedenen Instrumentalisten verwenden, um die Indios dazu zu bewegen, sich [dem Christentum] anzuschließen«. ${ }^{20}$ Von höchster Stelle wurde also die Anweisung erteilt, zur Missionierung nicht nur Gesang und Musikunterweisung, sondern auch eine Vielzahl von Instrumenten und Instrumentalisten einzusetzen.

Die Franziskaner im Mexico des 16. Jahrhunderts nutzten also Musik zur Annäherung, Kommunikation, zum Miteinander-Bekanntwerden und im Unter-

18 Brief von Zumárraga an Karl V. von 1540: »La experiencia muestra cuánto se edifican dello los naturales que son muy dados á la música, y los religiosos que oyen sus confesiones nos lo dicen, que más que por las predicaciones se convierten por la música, y los vemos venir de partes remotas para la oír.«In: Joaquín García Icazbalceta (Hrsg.), Don Fray Juan de Zumárraga, Primer Obispo y Arzobispo de México. Estudio Biográfico y Bibliográfico, México 1881, Anhang, Nr. 27, S. 137. Online verfügbar unter https://archive.org/stream/donfrayjuandezu00icazgoog\#page (Letzter Zugriff: 23.07.2015).

$19 »$ Pectora infidelium apprime commoventur eiusmodi ritibus, animique recentium Christianorum valde confirmantur \& capiuntur iis externis, cum adhuc sint parvuli \& quibus lacte opus sit non solido cibo.« Diego de Valadés und Esteban J.Palomera (Hrsg.), Fray Diego Valadés, OFM, Evangelizador Humanista de la Nueva España: Su Obra [Rhetorica Cristiana], México 1962, S. 227.

20 Online verfügbar unter http://www.biblioteca.tv/artman2/publish/1573_382/Ordenanzas_de_ Felipe_II_sobre_descubrimiento_nueva_1176.shtml (Letzter Zugriff: 23.07.2014); engl. Übersetzung in Urban Planning in North America, hrsg. von Daniel Garr, New York 1991, S. 30-31. $\mathrm{Zu}$ den ministriles altos y bajos siehe auch Reinhard Strohm, The Rise of European Music, Cambridge 1993, S. 300. 
richt, und sie berichteten über ihre Erfolge in der musikalischen Arbeit in Briefen, Berichten und Chroniken. Schon den Franziskanern scheint das sinnliche Moment von Musik wichtiger gewesen zu sein als das diskursive der christlichen Lehre; offensichtlich trauten auch sie schon der europäischen Musiksprache größeres Konversionspotential zu als der spanischen oder lateinischen Sprache der Heiligen Schrift. Die Idee von Mission durch Musik war auf jeden Fall im späten 16. Jahrhundert bereits fest etabliert.

Und sicherlich war dies den Jesuiten bekannt. Die erste Schrift eines Mitgliedes der Societas Jesu über Amerika, José de Acostas Historia natural y moral de las Indias wurde 1590 in Spanien gedruckt, nachdem der Verfasser sich sechzehn Jahre lang in Peru und Mittelamerika aufgehalten hatte. De Acosta schreibt zum Thema Mission durch Musik in einer Passage, die sich auf beide Regionen bezieht:

»Die Unsrigen, die unter ihnen [den Indigenen] arbeiten, haben probiert, die Grundlagen unseres heiligen Glaubens in ihre Art des Gesangs zu übersetzen, und sie ziehen großen Nutzen daraus, denn mit dem Gefallen am Gesang und den Liedern können sie [die Indigenen] ganze Tage zuhören und diese nachsingen, ohne zu ermüden. Sie haben auch Kompositionen und Lieder von uns in ihre Sprache übertragen, zum Beispiel octavas und canciones, romances und redondillas; und es ist ein Wunder, wie gut die Indios das aufnehmen, und wie sehr es ihnen gefällt: Das ist sicher ein gutes Mittel und für diese Leute sehr nützlich. $\ll^{21}$

Auch wenn sich nicht rekonstruieren lässt, auf welche Quellen sich der zu Anfang erwähnte Charlevoix in seiner Histoire du Paraguay stützte, klingt es bei ihm ähnlich - auch wenn er sich auf eine ganz andere Region bezieht.

»Dieses nun hat ihre Missionarien bewogen, in allen ihren Kirchen einen musicalischen Chor zu errichten; indem ihnen noch über dies die Erfahrung gelehrt hat, daß solches sehr viel dazu beyträgt, in ihnen eine Ehrfurcht zu erwecken, ihnen einen Geschmack an dem Gottesdienst beyzubringen, und ihnen die Lehren, die man ihnen vorgesagt, und die man in Gesänge gebracht hat, leichter begreiflich zu machen. $\ll^{22}$

Erst in den jesuitischen Texten der Spätzeit des Mission durch Musik-Narrativs, dem 18. Jahrhundert, werden seine Bestandteile überdeutlich: das imitatorische Talent, die wunderbare, angeblich natürliche musikalische Begabung der Indi-

21 José de Acosta, Historia natural y moral de las Indias, hrsg. von Edmundo O'Gorman, Mèxico 2006, VI/28, S. 354.

22 Charlevoix, Geschichte von Paraguay (wie Anm. 2), V, S. 359-360. 
genen und ihre extrem große Lernfähigkeit - die sich auch mit Homi Bhabhas Konzept von colonial mimicry erklären lässt ${ }^{23}$ - finden sich bereits bei Charlevoix:

»Sie begriffen, gleich als wenn es ihnen angeboren wäre, mit leichter Mühe, alle Künste, zu denen man sie anwies. [...] Sie verfertigen und spielen alle mögliche Instrumente in der Musik. ... sie haben von Natur ein scharfes Gehör, und einen ganz außerordentlich harmonischen Geschmack. Der Pater Cataneo, den ich schon oft angeführt habe, versichert, er habe ein Kind von zwölf Jahren, auf der Harfen die schwersten Stücke ohne Anstoß und sehr geschwind spielen sehen. Sie haben überdieß eine reine und helle Stimme, welches man, wie ich schon gesagt habe, den Wassern ihrer Flüsse zuschreibt. $\ll^{24}$

Während die Indigenen, so Charlevoix, zwar über einen »sehr stumpfen Verstand « verfügten und daher kaum eigenständig kreativ tätig werden konnten, verfügten sie über große Begabung und natürliche Talente im »Nachschöpfen«. Das scharfe Gehör, die reine und helle Stimme und der anßerordentliche harmonische Geschmack waren ihnen natürlich gegeben, und nur deswegen übertrafen die Indigenen die Europäer in vielen Handwerken und Künsten, die sie von ihnen gelernt hatten.

Oft wird auch deutlich, dass die natürliche Begabung, das imitatorische Talent und die große Lernfähigkeit der Indigenen nach Meinung der Missionare deren Erscheinungsbild widersprachen. Dies mündete bei einigen Autoren in einer herablassenden Exotisierung bzw Orientalisierung im Sinne Edward Saids ${ }^{25}$ bei vielen in den wohlbekannten Ideen von der zivilisatorischen Kraft der Musik. Die Idee der Zivilisierung war selbstverständlich neben der Konversion eines der Hauptziele der Mission, und auch dem Narrativ von Mission durch Musik war sie inhärent: denn wer nackt herumlief und weiterhin heidnische Rituale befolgte, konnte kaum glaubhaft europäische Musik singen. Wer dies doch tat, konnte nicht als Mensch wahrgenommen werden. So waren orientalisierende Äußerungen nicht nur eine Strategie zur Abwertung der in den Kolonien entstandenen Musikpraxis, sondern auch eine Taktik des Othering, um den indigenen Musikausübenden ihre

23 Homi Bhabha, »Of Mimicry and Man: The Ambivalence of Colonial Discourse«, in: Ders., The Location of Culture, London, New York 1994, S. 86.

24 Charlevoix, Geschichte von Paraguay (wie Anm. 3), V, S. 359-360.

25 Ich orientiere mich bei der Verwendung des Exotismus-Begriffs hier an Ralph Lockes sehr breiter Definition: [Musical exoticism] »is the process of evoking a place (people, social milieu) that is perceived as different from home by the people making and receiving the exoticist cultural product.« Ralph Locke, »A Broader View of Musical Exoticism«, in: The Journal of Musicology, 24/4 (2007), S. 477-521: S. 84. 
Menschlichkeit zu entziehen, wie in diesem Zitat eines jesuitischen Missionars aus dem frühen 18. Jahrhundert:

»Wie sollte einem Europäer nicht seltsam vorkommen zu sehen/ wie dass schwartze halb-nackende Barbarn die Lateinische Kirchen-Gesänger so künstlich singen/ und auf ihren Instrumenten in einem Dorff dermassen lieblich spihlen/ dass man ihnen in manchen Europäischen Städten mit Lust zuhorchen würde? «26

Von den Zeitgenossen des 16. und 17. Jahrhunderts ist nur ganz vereinzelt offene Kritik am etablierten Narrativ überliefert. EinZitat, das aber genau hier anschließt, stammt von einem Dominikaner, einem Angehörigen des Ordens, der für die Inquisition auch in Neuspanien zuständig war. Der in Sevilla geborene, in Mexico aufgewachsene Dominikaner Diego Durán warnte in seiner zwischen 1570 und 1581 verfassten Historia de las Indias de Nueva espana e Islas de la Tierra Firme vor Oberflächlichkeit und Verharmlosung, was als Kritik sowohl an den Franziskanern als auch an den Jesuiten verstanden werden muss. Er lenkte die Aufmerksamkeit seiner Leser auf die immer wieder von Missionaren arglos beobachteten »indianischen Tänze« und Gesänge und warnte:

»Es ist [bei diesen Tänzen] so, dass sie - während wir unwissend, sie aber eingeweiht in ihre alten Bräuche sind -, eine Gottheit darstellen, die sie vor unseren Augen auf ihre alte Weise feiern, und die Gesänge singen, die ihre Vorfahren ihnen zu diesem Zweck gelassen haben. $\ll^{27}$

Durán wandte sich gegen jedwede Duldung prächristlicher, indigener Lieder, denn diese würden gesungen, »wenn sie sehen, dass niemand, der sie versteht,

26 Brief von Ernst Steigmiller aus »Santa Fe di Bogotta«, 1724, in: Joseph Stöcklein, Der Neue Welt-Bott, 2/14, Augsburg, Graz 1729, Brief Nr. 324, S. 70. Siehe dazu auch Stephen Greenblatt, »Marvelous possessions«, in: The Greenblatt Reader, hrsg. von Michael Payne, Malden/MA, Oxford 2005, S. 93.

27 Diego Durán, Historia de las Indias de Nueva España e islas de la Tierra Firme, hrsg. von Ángel Maria Garibay Kintana, México 1967, S. 121-122: »12. Es que, nosotros ignorantes y ellos avisados en sus ritos antiguos, representan al ídolo, que están solemnizando delante de nosotros, a su modo antiguo, cantándole los cantares que sus viejos antiguos les dejaron. (...) 14. Digo que no se debe disimular ni permitir ande aquel indio allí representando su ídolo, y a lo demas cantores, sus idolatrías, cantos y lamentaciones, los cuales cantan mientras ven que no hay quien los entienda presente. Empero, en viendo que sale el que los entiende, mudan el canto y cantan el cantar que compusieron de San Francisco, con el Aleluya al cabo.« 
anwesend ist«. Sobald die Sänger bemerkten, dass jemand hinzu komme, »ändern sie das Lied und singen den Gesang, den die Franziskaner komponiert haben, mit dem Aleluya am Ende, um ihre Übeltaten zu überdecken. ${ }^{28}$

Dieses Zitat bildet zwar fast einen Einzelfall in der Missionsberichterstattung der Zeit, ist aber einzigartig in seiner Präzision und seinem Misstrauen gegenüber indigenem Christentum. Durán, der Dominikaner, war offensichtlich einer der wenigen Autoren, der dem ganzen Narrativ und seiner Einbettung in die jesuitische Mission schon im 16. Jahrhundert wahrhaftig misstraute.

Eine weniger grundsätzliche, mehr praktische Art des Misstrauens, ja sogar des musikalischen Misserfolgs zeichnet der Brief eines Missionars aus dem heutigen Südwesten Kolumbiens nach. Dieser ist einerseits durchaus als musikalische Bankrotterklärung zu sehen, vielleicht auch als Ärger darüber, dass in dieser Gegend die Mission durch Musik nicht so gut funktionierte wie anderswo:

»Massen unserer Amerikaner Spiel nicht viel besser herauskommt/ als wann Katzen und Hund miteinander zancken. Solches bestehet in liederlichen Leyren/ Härpfen/ Hackbretten/ ungestimmten Geigen/ und bruchhafften Trompeten/ welche so schön zusammen stimmen/ daß man aus dem Schall offtmal die Gattung des Instruments nicht ausnehmen kann/ ob es nemlich ein Seiten- oder Hornspiel seye/ und ob dasselbe geblasen/ gestrichen oder geschlagen werde. $\ll^{29}$

In diesem Zitat von 1724/1725 deutet sich schon an: Das Mission-durch-Musik-Narrativ war im 18. Jahrhundert, schon vor der Ausweisung der Jesuiten aus Spanisch- und Portugiesisch-Amerika 1759/1767 und der Auflösung des Ordens wenige Jahre später, an seinem natürlichen Ende angelangt. Besonders deutlich wird dies in satirischen Schriften wie Voltaires Candide ou l'optimisme von 1759: Hier reist die Hauptperson Candide nach Paraguay und trifft dort auf einen Jesuiten, der prahlerisch und verschwenderisch Hof hält, aus goldenen Schüsseln isst und aus Bergkristall-Gläsern trinkt. Später landen Candide und sein Diener Cacambo, der ein typischer indigener Jesuitenzögling ist,30 zufällig im mythenumwobenen El Dorado und treffen dort auf ein zufriedenes, glückliches Volk, das tiefgläubig ist und mit großer Selbstverständlichkeit nicht nur denselben christ-

\section{Ebda.}

29 Auszüge aus vier Briefen von Franz Xaver Zephyri aus Popayan/Latacunga (Provinz Quito), 1724/1725, in: Stöcklein, Neuer Welt-Bott (wie Anm. 26), II/13, Brief 283, S. 95.

$30 »$ Er war zu einem Viertel Spanier, Sohn eines Mestizen aus Tucuman. Er war Chorknabe, Mesner, Bootsmann, Mönch, Bote, Soldat und Lakai gewesen«, außerdem »Küchenjunge im [Jesuiten-] Kollegium in Asunción«, Voltaire, Candide ou l'optimisme, Paris 1759, hier zitiert aus der deutschen Übersetzung von Wolfgang Tschöke, München 2003, S. 48-49. 
lichen Gott anbetet wie der skeptische Candide, sondern auch noch befremdet auf dessen behutsames europäisches Nachfragen reagiert. Hier in El Dorado sind alle Träume der Europäer scheinbar Wirklichkeit geworden; das Christentum hat sich offensichtlich selbstverständlich verwurzelt. Auch was die Musikpraxis angeht, sind Dinge zur Realität geworden, die nicht nur in Europa unmöglich waren, sondern die auch niemand in der Neuen Welt für möglich gehalten hatte. Denn auf Candides Frage, wo denn die Priester des Volkes seien, antwortet ihm ein alter Mann: »Liebe Freunde, wir alle sind Priester; der König und alle Hausväter singen allmorgendlich feierliche Dankgesänge, und fünf- oder sechstausend Musiker begleiten sie. $\ll^{31}$

Nur zwei Jahre nach der Publikation von Charlevoix' Histoire du Paragnay wurde das Narrativ von Mission durch Musik in Voltaires Werk ad absurdum geführt und somit de facto beerdigt - auch wenn es Vielen bis heute scheinen mag, dass die Fabel von Orpheus und Amphion in Lateinamerika wahr geworden sei.

31 Siehe dazu auch den Artikel von Tomasz Jeż, »The Global Mission in the Music of Jesuit Drama«, in: Studies on a Global History of Music. A Balzan Musicology Project, hrsg. von Reinhard Strohm, Abingdon 2018 und T. Frank Kennedy, »Candide< and a boat«, in: The Jesuits. Cultures, sciences, and the arts, 1540-1773, hrsg. von John O’Malley u. a., Toronto 1999, S. 317-332. Bhabha, »Of Mimicry and Man« (wie Anm. 23). 